\title{
Rule violation errors are associated with right lateral prefrontal cortex atrophy in neurodegenerative disease
}

\author{
KATHERINE L. POSSIN,${ }^{1}$ SIMONA M. BRAMBATI, ${ }^{2}$ HOWARD J. ROSEN,${ }^{1}$ JULENE K. JOHNSON, ${ }^{1}$ \\ JUDY PA, ${ }^{1}$ MICHAEL W. WEINER, ${ }^{1}$ BRUCE L. MILLER,${ }^{1}$ AND JOEL H. KRAMER ${ }^{1}$ \\ ${ }^{1}$ Department of Neurology, University of California San Francisco, San Francisco, California \\ ${ }^{2}$ Centre de Recherche de l'Institut Universitaire de Gériatrie de Montréal, Montréal, Canada \\ (Received October 2, 2008; Final Revision January 3, 2009; AcCePted January 5, 2009)
}

\begin{abstract}
Good cognitive performance requires adherence to rules specific to the task at hand. Patients with neurological disease often make rule violation (RV) errors, but the anatomical basis for RV during cognitive testing remains debated. The present study examined the neuroanatomical correlates of RV errors made on tests of executive functioning in 166 subjects diagnosed with neurodegenerative disease or as neurologically healthy. Specifically, RV errors were voxelwisely correlated with gray matter volume derived from high-definition magnetic resonance images using voxel-based morphometry implemented in SPM2. Latent variable analysis showed that RV errors tapped a unitary construct separate from repetition errors. This analysis was used to generate factor scores to represent what is common among RV errors across tests. The extracted RV factor scores correlated with tissue loss in the lateral middle and inferior frontal gyri and the caudate nucleus bilaterally. When a more stringent control for global cognitive functioning was applied using Mini Mental State Exam scores, only the correlations with the right lateral prefrontal cortex (PFC) remained significant. These data underscore the importance of right lateral PFC in behavioral monitoring and highlight the potential of RV error assessment for identifying patients with damage to this region. (JINS, 2009, 15, 354-364.)
\end{abstract}

Keywords: Cognitive control, Neuropsychological assessment, Executive functioning, Voxel-based morphometry, Inhibition, Frontal lobe

\section{INTRODUCTION}

The interpretation of neuropsychological test performance has traditionally been based on overall achievement scores alone (Lezak et al., 2004; Reitan, 1993), although analyzing a patient's behavior en route to a solution (such as the errors they make) can also help in ascertaining the integrity of cognitive processes underlying neuropsychological test performance (Kaplan, 1988). Error scores derived from tests of executive functioning have shown clinical utility by improving differential diagnosis even after overall achievement scores have been taken into account (Carey et al., 2008; Culbertson et al., 2004; Kramer et al., 2003). In addition, executive error scores have shown anatomic specificity in their association with frontal lobe dysfunction (Alexander et al., 2007; Carey et al., 2008; Dao-Castellana

Correspondence and reprint requests to: Katherine L. Possin, 350 Parnassus Ste. 905, San Francisco, California 94143-1207. E-mail: kpossin@ memory.ucsf.edu et al., 1998; Stuss et al., 2001) and relationships to realworld behavior such as problems in goal-directed activities (Cahn-Weiner et al., 2002; Lawrence et al., 2004). Despite their potential clinical utility, executive errors are rarely incorporated into clinical trial assessments and are often given little emphasis in the differential diagnosis of neurodegenerative disease. One reason may be that the meaning of these errors is not well understood from a cognitive or neural standpoint.

One type of error that has shown particular clinical utility and anatomic specificity is rule violations (RVs). Tests of executive functioning require top-down cognitive control to direct responses according to task goals (Jurado \& Rosselli, 2007). When this limited-capacity control system fails, patients may not only produce fewer correct responses (resulting in a lower overall achievement score) but also produce more responses that violate test rules. Recently, Carey et al. (2008) demonstrated that RVs made by dementia patients on the Delis-Kaplan Executive Function System Tower Test correlated specifically with bilateral frontal lobe volumes, whereas overall achievement scores were 
less specific in their anatomic associations and correlated with both frontal and parietal brain volumes. Furthermore, they found that RVs successfully discriminated diagnostic subtypes as patients with frontotemporal dementia (FTD) made more RVs than patients with Alzheimer's disease (AD), whereas these patient groups did not differ in terms of overall achievement.

Previous studies of executive errors either have studied one specific test error in isolation (Carey et al., 2008) or have combined errors into a single composite score (Kramer et al., 2003) including repetitions and RVs, which may represent different types of cognitive failures (Possin et al., 2005). These approaches make it difficult to understand the meanings of similar errors made across tests, and so it is important that investigations into the associations of error scores consider which errors tap shared or distinct constructs. In addition, while executive errors have been associated with frontal lobe dysfunction, the frontal lobes are functionally heterogeneous, and it is not well understood which regions within the frontal lobes prevent these errors.

The goal of this study was to identify, in a large cohort of patients with neurodegenerative disease and neurologically healthy individuals, the brain regions where gray matter tissue loss was associated with a propensity to make RVs on tests of executive functioning. We used latent variable analysis to determine whether different RV error scores tap a unitary construct separate from repetition errors and to extract statistically what is common among RV errors made across tests. The extracted RV factor scores were then correlated with regional gray matter volumes. We hypothesized that $\mathrm{RV}$ errors, defined for this study as novel responses that violate test rules, tap a different cognitive construct from repetition errors, which are repetitions of a previously correct response. From an anatomical point of view, we hypothesized that RV errors would be associated with decreased volume in the lateral prefrontal cortex (PFC), which is widely understood to play a critical role in cognitive control (Bonelli \& Cummings, 2007; Courtney, 2004; MacDonald et al., 2000; Miller, 2000).

\section{METHOD}

\section{Subjects}

Magnetic resonance (MR) images were collected from a group of 166 subjects (age range 39-92 years, mean age 66 years, $S D=9$ ) composed of 74 females and 92 males recruited through and evaluated at the University of California San Francisco (UCSF) Memory and Aging Center. This heterogeneous sample was composed of patients who met established research criteria for one of seven diagnoses with variable effects on executive functioning (Kramer et al., 2006; Phukan et al., 2007; Pillon et al., 1995; Stokholm et al., 2006; Wittenberg et al., 2008) and patterns of brain atrophy (Boxer et al., 2006; Chang et al., 2005; Pa et al., in press; Rabinovici et al., 2007; Rosen et al., 2002). Including patients with these different clinical syndromes, as well as neurologically healthy controls (NCs), augmented the anatomical and behavioral variability of the sample and increased the power of the correlation analysis and the validity of our statistical model. Participants included subjects with diagnoses of mild cognitive impairment (MCI, $n=59$ ), FTD $(n=18), \mathrm{AD}(n=18)$, semantic dementia (SD, $n=14)$, corticobasal degeneration (CBD, $n=10)$, progressive supranuclear palsy $(n=3)$, amyotrophic lateral sclerosis $(n=3)$, and NC $(n=41)$. Patients were diagnosed using published diagnostic criteria (Brooks et al., 2000; Litvan et al., 1996, 2003; McKeith, 2006; McKhann et al., 1984; Neary et al., 1998) after a comprehensive evaluation including neurological history and examination, a caregiver interview, and a neuropsychological assessment that included the Mini Mental State Examination (Folstein et al., 1975 ) and tests of memory, executive function, language, visuospatial skills, and mood using a previously described standard protocol (Kramer et al., 2003). A research diagnosis of MCI was based on the following criteria: (1) complaint in one or more cognitive domains (i.e., memory, executive function, visuospatial, or language) reported by the subject, informant, or clinician; (2) report of a meaningful decline in one or more cognitive domains over a period of at least 1 year; (3) report of difficulty in the cognitive domain compared to age- and education-matched peers; (4) absence of dementia (American Psychiatric Association, 1994); and (5) the absence of other factors that could account for cognitive decline (e.g., major depression, substance abuse, hypothyroid). Of our MCI patients, 20 were classified as the dysexecutive subgroup (Pa et al., in press) and 25 met the Petersen criteria for MCI (Petersen et al., 1999). Demographic and clinical variables are reported in Table 1. Clinical Dementia Rating (CDR) Scale Scores are presented in this table as a measure of functional performance applicable to neurodegenerative disease (Morris, 1993). These scores are based on a semistructured interview of the patient and a reliable informant (e.g., family member) and are calculated on a scale from $0=$ normal to $3=$ severe dementia. ${ }^{\text {a }}$ All participants had a high-definition MR anatomical scan within 90 days of executive function assessment.

Exclusionary criteria for all subjects included presence of other neurological condition affecting behavior or cognition, longstanding Axis I psychiatric disorder, metabolic disorder or major organ dysfunction, alcohol abuse or dependence within 5 years, head trauma (with loss of consciousness for more than $30 \mathrm{~min}$ ), deteriorating cardiovascular disease, or prominent white matter disease. Patients with progressive nonfluent aphasia were excluded because these patients make errors on cognitive testing due to their language disturbance. The study was approved by the UCSF committee on human research. All subjects provided written informed consent before participating.

${ }^{a}$ Twenty-five of the 166 participants were not administered the CDR. These were $7 \mathrm{NC}, 14 \mathrm{MCI}, 2 \mathrm{AD}, 1 \mathrm{SD}$, and $1 \mathrm{CBD}$. 
Table 1. Demographic characteristics by diagnostic group

\begin{tabular}{lcccccc}
\hline \hline & $N$ & Age & Education & Proportion male & MMSE & CDR \\
\hline FTD & 18 & $62.3(8.9)$ & $16.7(2.2)$ & 0.78 & $26.2(2.7)$ & $1.2(0.5)$ \\
AD & 18 & $62.3(9.1)$ & $16.2(3.5)$ & 0.67 & $22.3(6.4)$ & $0.9(0.5)$ \\
PSP/CBD & 13 & $65.3(7.6)$ & $15.1(3.5)$ & 0.23 & $25.3(4.2)$ & $1.1(0.7)$ \\
SD & 14 & $62.7(7.3)$ & $15.5(3.2)$ & 0.57 & $25.1(3.4)$ & $0.5(0.3)$ \\
ALS & 3 & $58.1(11.6)$ & $15.7(.58)$ & 0.67 & $29.7(.58)$ & $0.7(0.3)$ \\
MCI & 59 & $69.1(9.3)$ & $17.8(2.2)$ & 0.61 & $28.7(1.5)$ & $0.5(0.1)$ \\
NC & 41 & $65.5(9.6)$ & $17.2(2.2)$ & 0.41 & $29.7(.68)$ & $0.0(0.1)$ \\
\hline \hline
\end{tabular}

Note. Values represent mean (SD). ALS, amyotrophic lateral sclerosis; PSP, progressive supranuclear palsy.

\section{RV and Repetition Error Assessment}

An executive functioning assessment was conducted on a separate day from the diagnostic evaluation and included the Trail Making, Letter Fluency, Design Fluency, and ColorWord Interference subtests from the Delis-Kaplan Executive Function System (Delis et al., 2001). Tabulated "RV Errors" were Trail Making Failure to Shift Errors, Letter Fluency Set-Loss Errors, Design Fluency Set-Loss Errors, and Color-Word Interference Uncorrected Errors. These errors were classified as RV errors because they are novel responses that violate the rules laid out to the examinee at the start of each test. Tabulated "Repetition Errors" were Letter Fluency Repetitions and Design Fluency Repetitions, which are previously emitted responses. The raw numbers of errors, rather than ratios of errors to correct responses, were used in the factor analysis. This decision was made based on our clinical experience that many patients with poor behavioral monitoring or inhibitory control, who show a propensity to make test errors, also emit a high number of responses within the time limit because they do not successfully monitor their accuracy and adjust their response speed accordingly. We did not want to deemphasize the errors made by these patients who are impaired in the construct that we were trying to measure. Operational definitions of the errors can be found in Table 2. The mean raw numbers of errors made by each diagnostic group are presented in Table 3, and the correlations among the errors are presented in Table 4.

\section{Neuroimaging Data}

Magnetic resonance imaging (MRI) scans were obtained on a 1.5-T Magnetom VISION system (Siemens, Iselin, NJ) at the San Francisco Veteran's Administration Hospital. A volumetric magnetization-prepared rapid gradient-echo MRI (repetition time, $10 \mathrm{~ms}$; echo time, $4 \mathrm{~ms}$; inversion time, $300 \mathrm{~ms}$ ) was used to obtain T1-weighted images of the entire brain, $15^{\circ}$ flip angle, coronal orientation perpendicular to the double spin-echo sequence, $1.0 \times 1.0 \mathrm{~mm}$ in-plane resolution, and $1.5 \mathrm{~mm}$ slab thickness.

\section{Voxel-Based Morphometry Preprocessing}

Voxel-based morphometry (VBM) is a technique for assessing voxel-by-voxel concentrations in brain tissue over the entire brain without bias to a priori identified structures (Ashburner \& Friston, 2000) and has been used to study the neuroanatomical correlates of cognition and behavior in many brain disorders including neurodegenerative disease (Amici et al., 2007; Rankin et al., 2006; Rosen et al., 2005). VBM analysis included two steps: spatial preprocessing (normalization, segmentation, Jacobian modulation, and smoothing) and statistical analysis. Both steps were implemented in the SPM2 software package (Wellcome Department of Imaging Neuroscience, London; http://www.fil. ion.ucl.ac.uk/spm) running on Matlab 6.5.1 (MathWorks, Natick, MA).

Table 2. Operational definitions of RV and repetition errors

\begin{tabular}{l}
\hline \hline RV errors \\
Trail Making Failure to Shift Errors: On the Number-Letter Switching Condition, lines drawn between two \\
elements from the same sequence (e.g., 2-3) rather than shifting between a number and a letter (e.g., 2-B). \\
Letter Fluency Set-Loss Errors: Responses not starting with the designated letter, names of people or places, \\
grammatical variants of the same word, numbers, and nonwords. \\
Design Fluency Set-Loss Errors: Responses that violate criterion rules. The most common types are designs that \\
contain more or fewer than four lines on any condition and designs that have a line that does not switch between \\
an empty dot and a filled dot on Condition 3: Switching. \\
Color-Word Interference Uncorrected Errors: Any errors made on the Inhibition or the Inhibition/Switching \\
Conditions that were not self-corrected. \\
Repetition errors \\
Letter Fluency Repetition Errors: Any repetition of a previously emitted response within a trial. \\
Design Fluency Repetition Errors: Any repetition of a previously drawn design within a condition.
\end{tabular}


Table 3. RV factor scores and raw error scores by diagnostic group

\begin{tabular}{lccccccc}
\hline \hline & FTD & AD & PSP/CBD & SD & ALS & MCI & NC \\
\hline RV factor scores & $1.0(1.3)$ & $.6(1.2)$ & $.5(.9)$ & $.0(1.0)$ & $-.7(.3)$ & $-.2(.8)$ & $-.6(.6)$ \\
Trailmaking Failure to Shift & $2.2(1.7)$ & $.8(1.1)$ & $1.4(1.8)$ & $.6(.9)$ & $.3(.6)$ & $.5(.7)$ & $.2(.5)$ \\
Color-Word Interference & $14.2(24.1)$ & $9.3(10.8)$ & $9.1(17.4)$ & $3.8(5.3)$ & $0.0(0.0)$ & $2.4(5.5)$ & $1.1(2.6)$ \\
Design Fluency Set-Loss & $3.3(3.8)$ & $3.4(2.6)$ & $3.8(3.4)$ & $2.9(5.4)$ & $1.0(0.0)$ & $2.1(2.0)$ & $2.0(2.8)$ \\
Letter Fluency Set-Loss & $1.8(1.6)$ & $2.3(3.8)$ & $.7(.9)$ & $1.6(1.8)$ & $.7(1.2)$ & $1.1(1.8)$ & $.7(2.6)$ \\
Design Fluency Repetitions & $5.7(5.5)$ & $1.4(2.1)$ & $2.2(2.5)$ & $2.8(3.5)$ & $2.7(1.2)$ & $4.1(5.2)$ & $2.2(2.4)$ \\
Letter Fluency Repetitions & $1.4(1.2)$ & $1.7(1.7)$ & $.9(.8)$ & $2.3(1.9)$ & $.3(.6)$ & $1.9(1.8)$ & $1.5(2.0)$ \\
\hline \hline
\end{tabular}

Values represent mean (s.d.).

MR images were preprocessed using an optimized method for the spatial normalization and segmentation of gray matter (Good et al., 2001b). All subject images were first spatially normalized and segmented in the Montreal Neurological Institute stereotaxic space to create normalized gray, white, and cerebrospinal fluid compartments, which were averaged across subjects to create normalization templates (Good et al., 2001b) and custom prior probability templates for tissue classification (Testa et al., 2004). The original images were then segmented in native space, and the resultant gray matter images were normalized to the study-specific gray matter template (same normalization parameters). The parameters obtained from the latter normalization were then applied to the original T1-weighted images, which were segmented again after normalization. Gray matter voxel values were multiplied by the Jacobian determinants derived from the spatial normalization step (Jacobian modulation) to preserve the initial volumes (Good et al., 2001a). Modulated gray matter images were then spatially smoothed with a $12-\mathrm{mm}$ full width at half maximum isotropic Gaussian kernel.

A covariate-only statistical model was used to correlate the RV factor scores and voxel-wise gray matter volumes with all subjects entered as a single group. We accounted for global level of atrophy by scaling each image by its total gray matter volume because we were interested in showing the specific regional associations with RVs that could not be explained by total gray matter differences. Age and sex were entered as nuisance covariates in the first analysis, and Mini Mental State Exam (MMSE) scores were additionally entered in the second analysis to correct for global cognitive impair- ment. The relationship between voxel values and RV scores was examined with a $-1 t$-contrast, assuming that decreased tissue content would be associated with an increased number of errors, and significance was determined using the theory of Gaussian fields. We accepted a statistical threshold of $p<$ .05 and corrected for multiple comparisons by using SPM family-wise error (FWE).

The same covariate-only statistical model was used to correlate repetition error scores and voxel-wise gray matter volumes. Age and sex were entered as nuisance covariates.

\section{RESULTS}

\section{Statistical Analysis of Errors}

Statistical analyses were performed with SPSS 15.0 for Windows (SPSS Inc., Chicago, IL). A principal components analysis was performed to test the a priori hypothesis that the four RV error scores tapped a shared construct separate from the two repetition error scores. First, the distributions of data collected on the four RV and two repetition error variables were examined and prepared for multivariate analysis. A square root transformation was performed on all six variables to reduce positive skew. Five univariate outliers were detected using a $z$ score cutoff of $3.29(p<.001)$. These four Color-Word Interference and one Letter Fluency Repetition scores were unattached to the rest of their distributions and were modified to one point higher than the next most extreme score (Tabachnick \& Fidell, 2001). Using Mahalanobis distance with $p<.001$, one multivariate outlier was identified

Table 4. Correlations among the six error scores

\begin{tabular}{lllllrl}
\hline \hline & TMFS & CWI & DFSL & LFSL & DFR & LFR \\
\hline TMFS & & $.39^{* *}$ & $.16^{*}$ & $.17^{*}$ & .08 & .05 \\
CWI & $.49^{* *}$ & & $.30^{* *}$ & $.20^{* *}$ & -.08 & .08 \\
DFSL & $.20^{*}$ & $.35^{* *}$ & & $.18^{*}$ & .12 & $.17^{*}$ \\
LFSL & $.23^{* *}$ & $.21^{* *}$ & $.24^{* *}$ & & -.01 & $.27^{* *}$ \\
DFR & .09 & -.002 & .08 & .001 & $.17^{* *}$ & .12 \\
LFR & .01 & .02 & $.17^{*}$ & $.24 *$ & $.17^{*}$ \\
\hline \hline
\end{tabular}

Note. Correlations among raw error scores are located in the bottom left and among transformed error scores in the top right. CWI, Color-Word Interference; DFSL, Design Fluency Set-Loss; DFR, Design Fluency Repetitions; LFR, Letter Fluency Repetitions; LFSL, Letter Fluency Set-Loss; TMFS, Trail Making Failure to Shift.

$* p<.05$.

$* * p<.01$ 
with a $z$ score value of 3.31 , and this subject was excluded from the analyses (Tabachnick \& Fidell, 2001). ${ }^{\mathrm{b}}$

Principal components extraction with varimax ${ }^{\mathrm{c}}$ rotation was performed through SPSS FACTOR on the six error measures for the sample of 166 subjects. The analysis yielded a two-component solution with the first component accounting for $30 \%$ of the variance and the second component accounting for $20 \%$ of the variance in the observed variables. Component loadings for each of the variables in the rotated solution can be seen in Table 5. Variables with loadings of .45 and above are interpreted (Comrey \& Lee, 1992). The four RV errors were found to load on Component 1, and the two repetition errors were found to load on Component 2. These findings provided support for our a priori hypothesis that RV errors tap a shared construct that is independent from repetition errors.

The principal components analysis was repeated with only the RV errors in order to generate factor scores for the neuroimaging analyses that would best represent the shared variance of these errors made across tests. The analysis yielded a one-component solution, accounting for $43 \%$ of the variance in the observed variables. Component loadings can be seen in Table 6. Factor scores for this component were derived using the regression approach in SPSS FACTOR. RV scores were not significantly correlated with age, $r=-.04, p=.60$, or total gray matter, $r=-.12, p=.25$. The scores significantly and negatively correlated with MMSE, $r=-.60, p<.01$. Table 3 shows the frequency of RV factor scores by patient group. Note that higher scores reflect a greater number of errors made.

\section{Neuroimaging Analyses}

VBM correlation analysis revealed a significant negative correlation between RV factor scores and gray matter volumes (less gray matter was associated with more RV errors) in the lateral PFC and the caudate nuclei (Table 7). The largest cluster of voxels that correlated with RV scores was in the posterior portion of the right inferior frontal gyrus (BA 44) and extending superiorly into the right middle frontal gyrus and posteriorly into the precentral gyrus. A significant association was also seen in the anterior portion of the right middle frontal gyrus (BA 46), the left inferior and middle frontal gyri extending posteriorly into the precentral gyrus (BA 44, 9), and in the dorsal caudate head bilaterally and extending into the body on the right. To verify whether the RV results could be ascribed, in part, to global cognitive effects, we reran the correlation analysis entering MMSE score as a nuisance variable. Only the correlations between the factor scores and gray matter volumes in the right inferior $(x=56, y=15, z=25, Z$ score $=4.71)$ and middle $(x=34$,

${ }^{\mathrm{b}}$ This subject was also removed from the report of subject characteristics in the Methods.

${ }^{\mathrm{c}}$ The solution did not change if the components were allowed to correlate.
Table 5. Component loadings of the six error scores

\begin{tabular}{lcc}
\hline \hline & $\begin{array}{c}\text { Component 1: } \\
\text { RVs }\end{array}$ & $\begin{array}{c}\text { Component 2: } \\
\text { repetitions }\end{array}$ \\
\hline Trail Making Failure to Shift & $\mathbf{. 7 0}$ & -.05 \\
Color-Word Interference & $\mathbf{. 8 2}$ & -.12 \\
Design Fluency Set-Loss & $\mathbf{. 5 3}$ & .33 \\
Letter Fluency Set-Loss & $\mathbf{. 4 7}$ & .38 \\
Design Fluency Repetitions & -.14 & $\mathbf{. 6 8}$ \\
Letter Fluency Repetitions & .16 & $\mathbf{. 7 5}$ \\
\hline
\end{tabular}

Note. Loadings for scores found to load on each component are represented in bold type.

$y=57, z=20, Z$ score $=4.69)$ frontal gyri were still significant at the threshold of $p<.05$, FWE corrected (Figure 1). ${ }^{\mathrm{d}}$

VBM correlation analyses were conducted with the transformed Design Fluency Repetition scores and with the transformed Verbal Fluency Repetition scores. These analyses were performed separately because the correlation between the repetition scores was small $(r=.17)$, and so analyses with a combined score could mask different relationships to gray matter volume. ${ }^{\mathrm{e}}$ No significant correlations were observed between Design Fluency Repetition errors and reduced gray matter volumes at the threshold of $p<.05$, FWE corrected. When we lowered our threshold on an exploratory basis to $p<.001$ uncorrected, Design Fluency Repetition errors were associated with reduced gray matter volumes in the bilateral anterior cingulate, bilateral medial superior frontal gyrus, and right orbitofrontal cortex. The Letter Fluency Repetition errors also did not correlate with reduced gray matter volumes at the threshold of $p<.05$, FWE corrected. At $p<.001$ uncorrected, Letter Fluency Repetition errors were associated with reduced gray matter volumes in the left fusiform gyrus, left parahippocampal gyrus, left hippocampus, and left inferior parietal lobule. Importantly, the gray matter correlates of the repetition error scores did not overlap with the gray matter correlates of the RV errors, even when no correction for multiple comparisons was applied to the significance threshold.

\section{DISCUSSION}

In an effort to better understand the meanings of RV errors from a neuroanatomical standpoint, we correlated factor

${ }^{\mathrm{d}}$ In order to evaluate whether outliers on the MMSE or the RV factor scores may have impacted the results, this analysis was rerun after removing the four subjects with univariate outliers on one or both of these measures (using a $z$ score cutoff of 3.29). The correlation between the factor scores and gray matter volumes in the right middle frontal gyrus was still significant at the threshold of $p<.05$, FWE corrected $(x=36, y=58, z=19$, $Z$ score $=4.63)$. The correlation with voxels in the right inferior frontal gyrus was no longer significant at FWE correction $(x=54, y=17, z=25$, $Z$ score $=4.26$ ) but was highly significant when uncorrected for multiple comparisons, $p<.001$. This follow-up analysis indicates that the results were not substantially impacted by outlier scores.

eA combined Design and Letter Repetition Error factor score also showed no significant correlations with gray matter volume, $p<.05$, FWE corrected. 
Table 6. Component loadings of the RV scores

\begin{tabular}{lc}
\hline \hline & Component: RVs \\
\hline Trail Making Failure to Shift & .68 \\
Color-Word Interference & .78 \\
Design Fluency Set-Loss & .53 \\
Letter Fluency Set-Loss & .61 \\
\hline \hline
\end{tabular}

scores derived from four types of these errors made across tests of executive functioning with voxel-wise gray matter volumes in a mixed sample of 166 subjects who had MCI or dementia or were neurologically healthy. We found that a propensity to make these errors corresponded to reduced gray matter volume in the lateral inferior and middle frontal gyri and the caudate nuclei bilaterally. The correlations with right lateral PFC remained significant even after MMSE scores were included as a covariate in the model, suggesting that the relationship between atrophy in this region and a propensity to make RV errors cannot be explained by global cognitive impairment. No correlations were observed with nonfrontal regions (temporal, parietal, or occipital cortex). These results are consistent with previous findings that executive function test RV errors are associated with frontal lobe dysfunction (Alexander et al., 2007; Carey et al., 2008; Cato et al., 2004; Dao-Castellana et al., 1998; Stuss et al., 2001) and further demonstrate that the right lateral frontal lobe in particular may be critical for preventing these errors. Repetition errors were shown to tap a different construct from RV errors because they loaded independently in the principal components analysis and did not correlate with lateral prefrontal or caudate nuclei volumes.

The present study's findings highlight the importance of the right lateral PFC in behavioral monitoring and suggest that the cognitive processes underlying this behavioral monitoring may be multifactorial. On tests of executive functioning including the four used in this study, a subject is required to maintain the test rules online in working memory, respond according to the rules, and inhibit behavior that would violate the rules. For example, on the Trail Making Test, the patient must remember to shift back and forth between sequencing numbers and letters while inhibiting the overlearned tendency to sequence numbers only or letters only. On the Color-Word Interference Test, the patient must remember to name the color ink that the color name is printed in and also inhibit reading the word. On tests of fluency, the patient must remember what types of words to name or what types of designs to draw and respond only according to those specifications. Inhibitory demands are particularly challenged by all four of these tests because patients must generate responses within time constraints. Based on this conceptualization of what is common among these RV errors, these errors may result from a breakdown in the maintenance of task-relevant information or in the suppression of taskirrelevant information or responses. Consistent with this interpretation, the right middle and inferior frontal gyri are engaged on tasks that use working memory to suppress automatic behavior (Aron et al., 2004; Buchsbaum et al., 2005; Simmonds et al., 2008).

Working memory and inhibitory control may be interdependent processes that are mediated by the lateral PFC. Lesions involving the middle frontal gyrus have been shown to impair both the maintenance of working memory representations and the suppression of reflexive saccades (Funahashi et al., 1993; Pierrot-Deseilligny et al., 2003). Dual-task paradigms have been used to show that when working memory demands are increased, we are more susceptible to the processing of irrelevant information and to behavioral interference (Bunge et al., 2001; Hester et al., 2004; Mitchell et al., 2002). Using functional MRI, de Fockert et al. (2001) showed that performance on a visual selective attention task that required subjects to ignore faces was impaired when subjects had to maintain a high versus low verbal working memory load during the delay and that maintenance of a higher load was associated with greater activation of lateral PFC as well as of visual areas involved in face processing (Gazzaley et al., 2005, 2007). Taken together, this body of research suggests that the enhancement of taskrelevant representations in working memory and the suppression of task-irrelevant representations or behavior are related processes mediated, in part, by the lateral PFC. There

Table 7. Regions where RV factor scores negatively correlated with gray matter tissue volume in the analysis without MMSE entered as a nuisance variable

\begin{tabular}{lcccc}
\hline \hline Brain region (BA) & $x$ & $y$ & $z$ & $Z$ score \\
\hline R inferior frontal gyrus (44) & 51 & 15 & 25 & 5.23 \\
R precentral gyrus/inferior frontal gyrus (44) & 46 & 9 & 30 & 5.11 \\
R middle frontal gyrus (44/6) & 44 & 9 & 40 & 5.03 \\
R middle frontal gyrus (46) & 36 & 58 & 21 & 4.59 \\
L precentral gyrus/inferior frontal gyrus (44) & -42 & 11 & 33 & 5.28 \\
L precentral gyrus/middle frontal gyrus (9) & -45 & 11 & 46 & 4.71 \\
R caudate nucleus & 11 & 14 & 8 & 4.60 \\
L caudate nucleus & -12 & 15 & 6 & 4.62 \\
\hline \hline
\end{tabular}

Note. Voxels are reported in Montreal Neurological Institute coordinates ( $x$ : right-left, $y$ : anterior-posterior, $z$ : superior-inferior) and are corrected for FWE across the whole brain at a significance level of $p<.05$. 


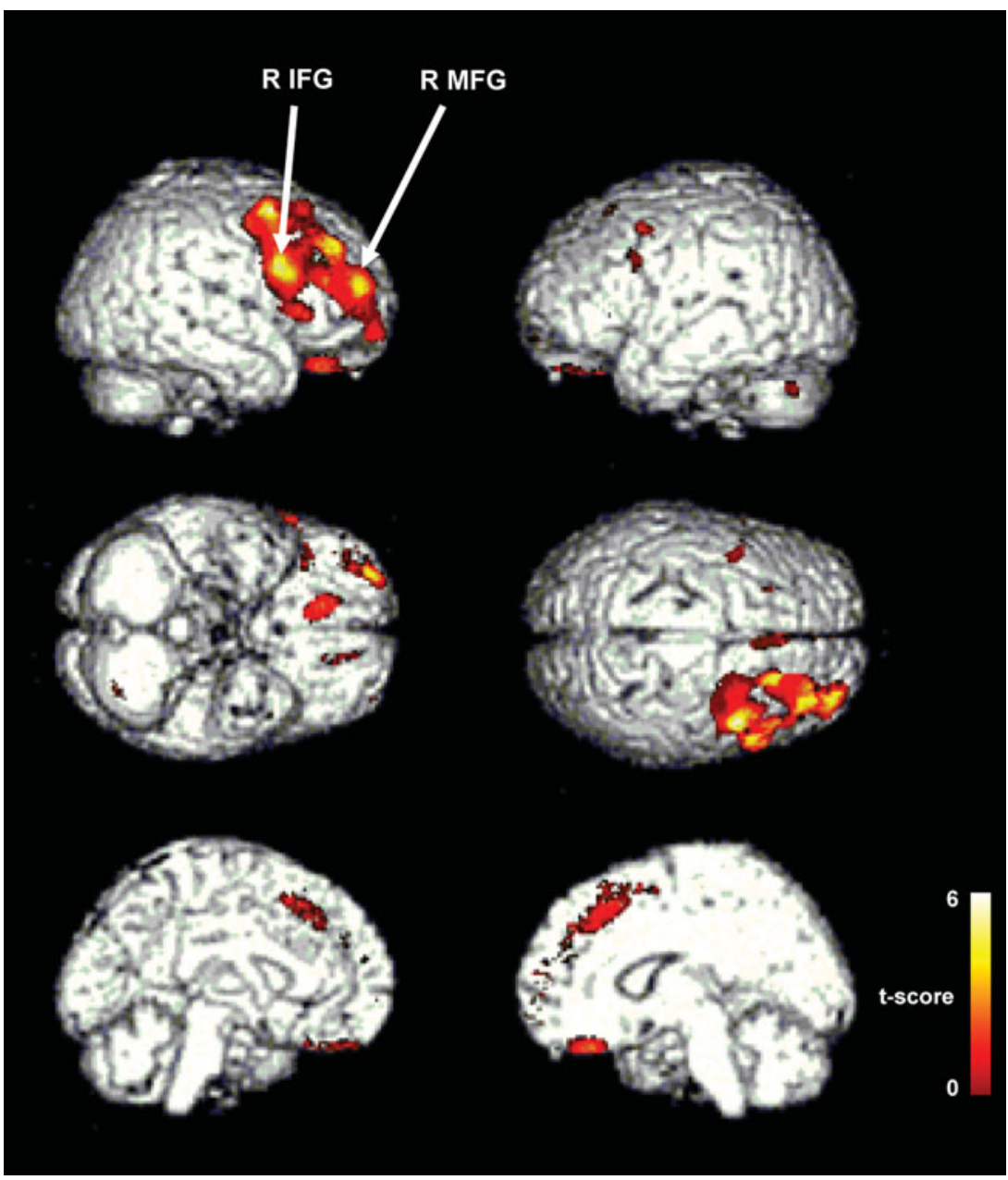

Fig. 1. Regions of gray matter tissue loss associated with RV factor scores after correcting for MMSE, $p<.001$ uncorrected. Arrows point to regions in right inferior frontal gyrus (IFG) and middle frontal gyrus (MFG) that are significant with FWE correction.

is also some evidence that the caudate nucleus is important for working memory (Possin et al., 2008; Sullivan \& Sagar, 1991), inhibitory attention (Fielding et al., 2006; Filoteo et al., 1997; Possin et al., in press), and the suppression of automatic behavioral responses (Chan et al., 2005; Henik et al., 1993), which suggests that the broader lateral prefrontalstriatal network may play a role in behavioral monitoring.

The present results suggest that atrophy in both the right and left lateral PF-striatal networks is associated with a propensity to make RV errors, but the most salient effect was with the right lateral PFC, which survived a correction for global cognitive impairment. While the representation of rules in working memory appears to rely on both right and left lateral PFC (Courtney, 2004; Wallis et al., 2001), there is some evidence that the right lateral PFC, particularly involving the inferior frontal gyrus, is dominant when the rules must be applied to suppress automatic behavior (Aron et al., 2004; Buchsbaum et al., 2005; Chikazoe et al., 2007; Clark et al., 2007). Engagement of the frontal cortex during the Go/No-go response inhibition paradigm differs by studies such that a right-lateralized network is activated only when multiple stimulus-response associa- tions have to be maintained or updated to guide performance (Simmonds et al., 2008). Right lateral PFC has also been shown to be engaged during the inhibition condition (name the color ink, don't read the word) but not the word reading condition of a Stroop task and exclusively during the cue condition when the inhibitory rule is updated in working memory (Donohue et al., 2008). These studies, taken together with the present results, indicate that the right lateral PFC may be more engaged than the left when working memory for current task rules is necessary to guide response inhibition.

Patients with neurodegenerative disease or lesions involving the right frontal lobe more frequently violate social rules than their counterparts with left frontal lobe damage (Mychack et al., 2001; Tranel et al., 2002). Mychack et al. (2001) showed that 11 out of 12 patients with right-sided FTD had aggressive, antisocial, or other socially undesirable behavior presenting as an early symptom, while only 2 of 19 patients with left-sided FTD showed these behaviors. Rosen et al. (2005) examined the neuroanatomical correlates of behavioral abnormalities in neurodegenerative disease using VBM and found that behavioral abnormalities were associated 
with tissue loss in right PFC involving predominantly medial and ventral regions as well as the lateral middle frontal gyrus. Behavioral disinhibition had a unique association with the right subgenual cingulate gyrus in the ventromedial PFC. Taken together with the present study, the right PFC appears to be generally involved in the inhibition of behavior that violates rules. When following the rules does not rely heavily on working memory but does require an adaptation to changing or conflicting reward values, ventromedial PFC is engaged (Viskontas et al., 2007). When following the rules does rely heavily on working memory because the rules are novel, complex, or changing over time, as is the case during executive function assessment, the lateral PFC is recruited to prevent these errors.

A heterogeneous patient sample was selected for this study to augment anatomical and behavioral variability and increase the validity of our statistical model. The neurodegenerative disease diagnoses selected for this study vary in their impact on executive functioning and are associated with different patterns of gray matter atrophy. This variability increased our power to find a relationship between the error measures and gray matter volume. A limitation of studying a heterogeneous sample, however, is that the results may obscure the specific cognitive deficits and neuroanatomical correlates in a homogeneous subgroup (Delis et al., 2003), in which the four measures of RVs might not all load on the same component. In addition, it is quite likely that the individual error scores manifest heterogeneous underlying mechanisms in addition to a common construct, as reflected by the low-to-moderate correlations among the individual RV error scores (Table 4). For example, Failure to Shift errors on Trail Making may represent a failure to shift set, whereas intrusions on fluency tasks may represent a failure to maintain set. If a patient group were impaired in set shifting but not set maintenance, then one would expect these error types to load on different components. The RV factor scores also could have different neuroanatomical correlates within one diagnostic group that is masked by including that group in the heterogeneous sample. In order to determine whether the relationship we observed holds in each of these subgroups, particularly in subgroups without prominent lateral prefrontal atrophy, one would need a very large sample.

Out of the comprehensive battery of tests that were administered for diagnostic purposes, MMSE was the best choice as a single measure of global cognitive functioning because it samples orientation, memory, visuospatial skills, and language skills and because it is known to decline with neurodegenerative disease progression (Teri et al., 1990; Rascovsky et al., 2005). The MMSE has limitations for assessing general cognition in neurodegenerative disease, however. This measure places minimal demands on executive functioning (Binetti et al., 1996). For the purpose of this study, this criticism represented a strength because we were able to provide some correction for global cognition while removing only a minimal amount of meaningful variance associated with our executive function error scores. Another criticism of the MMSE is that it is biased toward an assessment of language skills (Osher et al., 2007). This bias may have removed more lefthemisphere variance than right-hemisphere variance when MMSE was entered as a nuisance variable. Thus, while we can be most confident that the relationship between right lateral PFC and RV errors is independent of global cognitive impairment, it is possible that this laterality effect is driven, in part, by the MMSE's language bias.

Although rarely emphasized in neuropsychological assessment, the evaluation of error types has shown potential for increasing the accuracy of neurodegenerative disease differential diagnosis (Carey et al., 2008; Culbertson et al., 2004; Kramer et al., 2003) and for predicting real-world behavior (Cahn-Weiner et al., 2002; Lawrence et al., 2004). We found that RV errors elicited across tests of executive functioning were associated with atrophy in the lateral PFC and the caudate nucleus bilaterally and were not associated with atrophy in posterior cortex. Regions in right middle and inferior frontal gyri, which remained significant after correcting for global cognitive functioning, may be particularly critical for using working memory to guide the inhibition of automatic responses. The use of RV errors in neuropsychological assessment may help in identifying patients with right lateral PFC dysfunction particularly when their propensity to make these errors exceeds what would be expected by their global cognitive impairment.

\section{ACKNOWLEDGMENTS}

This work was supported by the National Institute on Aging grants AG22983, P50-AG05142, and AG022538 and the State of California Alzheimer's Disease Research Center of California grant 01154-20. The information in this article has never before been published either electronically or in print.

\section{REFERENCES}

Alexander, M.P., Stuss, D.T., Picton, T., Shallice, T., \& Gillingham, S. (2007). Regional frontal injuries cause distinct impairments in cognitive control. Neurology, 68(18), 1515-1523.

American Psychiatric Association. (1994). Diagnostic and Statistical Manual of Mental Disorders-(DSM-IV). Washington, D.C.

Amici, S., Brambati, S.M., Wilkins, D.P., Ogar, J., Dronkers, N.L., Miller, B.L., \& Gorno-Tempini, M.L. (2007). Anatomical correlates of sentence comprehension and verbal working memory in neurodegenerative disease. Journal of Neuroscience, 27(23), 6282-6290.

Aron, A.R., Robbins, T.W., \& Poldrack, R.A. (2004). Inhibition and the right inferior frontal cortex. Trends in Cognitive Sciences, 8(4), 170-177.

Ashburner, J. \& Friston, K.J. (2000). Voxel-based morphometryThe methods. NeuroImage, 11(6 Pt 1), 805-821.

Binetti, G., Magni, E., Padovani, A., Cappa, S.F., Bianchetti, A., \& Trabucchi, M. (1996). Executive dysfunction in early Alzheimer's disease. Journal of Neurology, Neurosurgery, and Psychiatry, 60(1), 91-93.

Bonelli, R.M. \& Cummings, J.L. (2007). Frontal-subcortical circuitry and behavior. Dialogues in Clinical Neuroscience, 9(2), 141-151. 
Boxer, A.L., Geschwind, M.D., Belfor, N., Gorno-Tempini, M.L., Schauer, G.F., Miller, B.L., Weiner, M.W., \& Rosen, H.J. (2006). Patterns of brain atrophy that differentiate corticobasal degeneration syndrome from progressive supranuclear palsy. Archives of Neurology, 63(1), 81-86.

Brooks, B.R., Miller, R.G., Swash, M., \& Munsat, T.L. (2000). El Escorial revisited: Revised criteria for the diagnosis of amyotrophic lateral sclerosis. Amyotrophic Lateral Sclerosis and Other Motor Neuron Disorders, 1(5), 293-299.

Buchsbaum, B.R., Greer, S., Chang, W.L., \& Berman, K.F. (2005). Meta-analysis of neuroimaging studies of the Wisconsin cardsorting task and component processes. Human Brain Mapping, 25(1), 35-45.

Bunge, S.A., Ochsner, K.N., Desmond, J.E., Glover, G.H., \& Gabrieli, J.D. (2001). Prefrontal regions involved in keeping information in and out of mind. Brain, 124(Pt 10), 2074-2086.

Cahn-Weiner, D.A., Boyle, P.A., \& Malloy, P.F. (2002). Tests of executive function predict instrumental activities of daily living in community-dwelling older individuals. Applied Neuropsychology, 9(3), 187-191.

Carey, C.L., Woods, S.P., Damon, J., Halabi, C., Dean, D., Delis, D.C., Miller, B.L., \& Kramer, J.H. (2008). Discriminant validity and neuroanatomical correlates of rule monitoring in frontotemporal dementia and Alzheimer's disease. Neuropsychologia, 46(4), 1081-1087.

Cato, M.A., Delis, D.C., Abildskov, T.J., \& Bigler, E. (2004). Assessing the elusive cognitive deficits associated with ventromedial prefrontal damage: A case of a modern-day Phineas Gage. Journal of the International Neuropsychological Society, 10(3), 453-465.

Chan, F., Armstrong, I.T., Pari, G., Riopelle, R.J., \& Munoz, D.P. (2005). Deficits in saccadic eye-movement control in Parkinson's disease. Neuropsychologia, 43(5), 784-796.

Chang, J.L., Lomen-Hoerth, C., Murphy, J., Henry, R.G., Kramer, J.H., Miller, B.L., \& Gorno-Tempini, M.L. (2005). A voxelbased morphometry study of patterns of brain atrophy in ALS and ALS/FTLD. Neurology, 65(1), 75-80.

Chikazoe, J., Konishi, S., Asari, T., Jimura, K., \& Miyashita, Y. (2007). Activation of right inferior frontal gyrus during response inhibition across response modalities. Journal of Cognitive Neuroscience, 19(1), 69-80.

Clark, L., Blackwell, A.D., Aron, A.R., Turner, D.C., Dowson, J., Robbins, T.W., \& Sahakian, B.J. (2007). Association between response inhibition and working memory in adult ADHD: A link to right frontal cortex pathology? Biological Psychiatry, 61(12), 1395-1401.

Comrey, A.L. \& Lee, H.B. (1992). A first course in factor analysis. Hillsdale, NJ: Lawrence Erlbaum Associates.

Courtney, S.M. (2004). Attention and cognitive control as emergent properties of information representation in working memory. Cognitive, Affective \& Behavioral Neuroscience, 4(4), 501-516.

Culbertson, W.C., Moberg, P.J., Duda, J.E., Stern, M.B., \& Weintraub, D. (2004). Assessing the executive function deficits of patients with Parkinson's disease: Utility of the Tower of London-Drexel. Assessment, 11(1), 27-39.

Dao-Castellana, M.H., Samson, Y., Legault, F., Martinot, J.L., Aubin, H.J., Crouzel, C., Feldman, L., Barraucand, D., Rancurel, G., Feline, A., \& Syrota, A. (1998). Frontal dysfunction in neurologically normal chronic alcoholic subjects: Metabolic and neuropsychological findings. Psychological Medicine, 28(5), 1039-1048.

de Fockert, J.W., Rees, G., Frith, C.D., \& Lavie, N. (2001). The role of working memory in visual selective attention. Science, 291(5509), 1803-1806.
Delis, D.C., Jacobson, M., Bondi, M.W., Hamilton, J.M., \& Salmon, D.P. (2003). The myth of testing construct validity using factor analysis or correlations with normal or mixed clinical populations: Lessons from memory assessment. Journal of the International Neuropsychological Society, 9(6), 936-946.

Delis, D., Kaplan, E.B., \& Kramer, J. (2001). The Delis-Kaplan Executive Function System. San Antonio, TX: The Psychological Corporation.

Donohue, S.E., Wendelken, C., \& Bunge, S.A. (2008). Neural correlates of preparation for action selection as a function of specific task demands. Journal of Cognitive Neuroscience, 20(4), 694-706.

Fielding, J., Georgiou-Karistianis, N., \& White, O. (2006). The role of the basal ganglia in the control of automatic visuospatial attention. Journal of the International Neuropsychological Society, 12(5), 657-667.

Filoteo, J.V., Delis, D.C., Salmon, D.P., Demadura, T., Roman, M.J., \& Shults, C.W. (1997). An examination of the nature of attentional deficits in patients with Parkinson's disease: Evidence from a spatial orienting task. Journal of the International Neuropsychological Society, 3(4), 337-347.

Folstein, M.F., Folstein, S.E., \& McHugh, P.R. (1975). "Minimental state". A practical method for grading the mental state of patients for the clinician. Journal of Psychiatric Research, 12, 189-198.

Funahashi, S., Bruce, C.J., \& Goldman-Rakic, P.S. (1993). Dorsolateral prefrontal lesions and oculomotor delayed-response performance: Evidence for mnemonic "scotomas". Journal of Neuroscience, 13(4), 1479-1497.

Gazzaley, A., Cooney, J.W., Rissman, J., \& D'Esposito, M. (2005). Top-down suppression deficit underlies working memory impairment in normal aging. Nature Neuroscience, $8(10)$, $1298-1300$

Gazzaley, A., Rissman, J., Cooney, J., Rutman, A., Seibert, T., Clapp, W., \& D'Esposito, M. (2007). Functional interactions between prefrontal and visual association cortex contribute to top-down modulation of visual processing. Cerebral Cortex, 17(Suppl 1), i125-i135.

Good, C.D., Johnsrude, I., Ashburner, J., Henson, R.N., Friston, K.J., \& Frackowiak, R.S. (2001a). Cerebral asymmetry and the effects of sex and handedness on brain structure: A voxel-based morphometric analysis of 465 normal adult human brains. NeuroImage, 14(3), 685-700.

Good, C.D., Johnsrude, I.S., Ashburner, J., Henson, R.N., Friston, K.J., \& Frackowiak, R.S. (2001b). A voxel-based morphometric study of ageing in 465 normal adult human brains. NeuroImage, 14(1 Pt 1), 21-36.

Henik, A., Singh, J., Beckley, D.J., \& Rafal, R.D. (1993). Disinhibition of automatic word reading in Parkinson's disease. Cortex, 29(4), 589-599.

Hester, R., Murphy, K., \& Garavan, H. (2004). Beyond common resources: The cortical basis for resolving task interference. NeuroImage, 23(1), 202-212.

Jurado, M.B. \& Rosselli, M. (2007). The elusive nature of executive functions: A review of our current understanding. Neuropsychology Review, 17(3), 213-233.

Kaplan, E. (1988). A process approach to neuropsychological assessment. In T. Boll \& B. Bryant (Eds.), Clinical neuropsychology and brain function: Research, measurement, and practice (pp. 129-167). Washington, DC: American Psychological Association.

Kramer, J.H., Jurik, J., Sha, S.J., Rankin, K.P., Rosen, H.J., Johnson, J.K., \& Miller, B.L. (2003). Distinctive neuropsychological 
patterns in frontotemporal dementia, semantic dementia, and Alzheimer disease. Cognitive and Behavioral Neurology, 16(4), 211-218.

Kramer, J.H., Nelson, A., Johnson, J.K., Yaffe, K., Glenn, S., Rosen, H.J., \& Miller, B.L. (2006). Multiple cognitive deficits in amnestic mild cognitive impairment. Dementia and Geriatric Cognitive Disorders, 22(4), 306-311.

Lawrence, V., Houghton, S., Douglas, G., Durkin, K., Whiting, K., \& Tannock, R. (2004). Executive function and ADHD: A comparison of children's performance during neuropsychological testing and real-world activities. Journal of Attention Disorders, 7(3), 137-149.

Lezak, M.D., Howieson, D.B., Loring, D.W., Loring, D.W., Hannay, H.J., \& Fischer, J.S. (2004). Neuropsychological assessment (4th ed.). Oxford: Oxford University Press.

Litvan, I., Agid, Y., Calne, D., Campbell, G., Dubois, B., Duvoisin, R.C., Goetz, C.G., Golbe, L.I., Grafman, J., Growdon, J.H., Hallett, M., Jankovic, J., Quinn, N.P., Tolosa, E., \& Zee, D.S. (1996). Clinical research criteria for the diagnosis of progressive supranuclear palsy (Steele-Richardson-Olszewski syndrome): Report of the NINDS-SPSP international workshop. Neurology, 47(1), 1-9.

Litvan, I., Bhatia, K.P., Burn, D.J., Goetz, C.G., Lang, A.E., McKeith, I., Quinn, N., Sethi, K.D., Shults, C., Wenning, G.K., \& Movement Disorders Society Scientific Issues Committee. (2003). Movement Disorders Society Scientific Issues Committee report: SIC Task Force appraisal of clinical diagnostic criteria for Parkinsonian disorders. Movement Disorders, 18(5), 467-486.

MacDonald, A.W.3rd, Cohen, J.D.,, Stenger, V.A., \& Carter, C.S. (2000). Dissociating the role of the dorsolateral prefrontal and anterior cingulate cortex in cognitive control. Science, 288(5472), $1835-1838$.

McKeith, I.G. (2006). Consensus guidelines for the clinical and pathologic diagnosis of dementia with Lewy bodies (DLB): Report of the Consortium on DLB International Workshop. Journal of Alzheimer's Disease, 9(3 Suppl), 417-423.

McKhann, G., Drachman, D., Folstein, M., Katzman, R., Price, D., \& Stadlan, E.M. (1984). Clinical diagnosis of Alzheimer's disease: Report of the NINCDS-ADRDA Work Group under the auspices of Department of Health and Human Services Task Force on Alzheimer's Disease. Neurology, 34(7), 939-944.

Miller, E.K. (2000). The prefrontal cortex and cognitive control. Nature Reviews. Neuroscience, 1(1), 59-65.

Mitchell, J.P., Macrae, C.N., \& Gilchrist, I.D. (2002). Working memory and the suppression of reflexive saccades. Journal of Cognitive Neuroscience, 14(1), 95-103.

Morris, J.C. (1993). The Clinical Dementia Rating (CDR): Current version and scoring rules. Neurology, 43(11), 2412-2414.

Mychack, P., Kramer, J.H., Boone, K.B., \& Miller, B.L. (2001). The influence of right frontotemporal dysfunction on social behavior in frontotemporal dementia. Neurology, 56(11 Suppl 4), S11-S15.

Neary, D., Snowden, J.S., Gustafson, L., Passant, U., Stuss, D., Black, S., Freedman, M., Kertesz, A., Robert, P.H., Albert, M., Boone, K., Miller, B.L., Cummings, J., \& Benson, D.F. (1998). Frontotemporal lobar degeneration: A consensus on clinical diagnostic criteria. Neurology, 51(6), 1546-1554.

Osher, J.E., Wicklund, A.H., Rademaker, A., Johnson, N., \& Weintraub, S. (2007). The mini-mental state examination in behavioral variant frontotemporal dementia and primary progressive aphasia. American Journal of Alzheimer's Disease and Other Dementia's, 22(6), 468-473.
Pa, J., Boxer, A.L., Freeman, K., Kramer, J.H., Miller, B.L., Chao, L.L., Gazzaley, A., Weiner, M.W., Neuhaus, J., \& Johnson, J.K. (in press). Clinical-neuroimaging characteristics of dysexecutive mild cognitive impairment. Annals of Neurology.

Petersen, R.C., Smith, G.E., Waring, S.C., Ivnik, R.J., Tangalos, E.G., \& Kokmen, E. (1999). Mild cognitive impairment: Clinical characterization and outcome. Archives of Neurology, 56(3), 303-308.

Phukan, J., Pender, N.P., \& Hardiman, O. (2007). Cognitive impairment in amyotrophic lateral sclerosis. Lancet Neurology, 6(11), 994-1003.

Pierrot-Deseilligny, C., Muri, R.M., Ploner, C.J., Gaymard, B., Demeret, S., \& Rivaud-Pechoux, S. (2003). Decisional role of the dorsolateral prefrontal cortex in ocular motor behaviour. Brain, 126(Pt 6), 1460-1473.

Pillon, B., Blin, J., Vidailhet, M., Deweer, B., Sirigu, A., Dubois, B., \& Agid, Y. (1995). The neuropsychological pattern of corticobasal degeneration: Comparison with progressive supranuclear palsy and Alzheimer's disease. Neurology, 45(8), 1477-1483.

Possin, K.L., Filoteo, J.V., Roesch, S.C., Zizak, V., Rilling, L.M., \& Davis, J.D. (2005). Is a perseveration a perseveration? An evaluation of cognitive error types in patients with subcortical pathology. Journal of Clinical and Experimental Neuropsychology, 27(8), 953-966.

Possin, K.L., Filoteo, J.V., Song, D.D., \& Salmon, D.P. (2008). Spatial and object working memory deficits in Parkinson's disease are due to impairment in different underlying processes. Neuropsychology, 22(5), 585-595.

Possin, K.L., Filoteo, J.V., Song, D.D., \& Salmon, D.P. (in press). Space-based but not object-based inhibition of return is impaired in Parkinson's disease. Neuropsychologia.

Rabinovici, G.D., Seeley, W.W., Kim, E.J., Gorno-Tempini, M.L., Rascovsky, K., Pagliaro, T., Allison, S.C., Halabi, C., Kramer, J.H., Johnson, J.K., Weiner, M.W., Forman, M.S., Trojanowski, J.Q., Dearmond, S.J., Miller, B.L., \& Rosen, H.J. (2007). Distinct MRI atrophy patterns in autopsy-proven Alzheimer's disease and frontotemporal lobar degeneration. American Journal of Alzheimer's Disease and Other Dementias, 22(6), 474-488.

Rankin, K.P., Gorno-Tempini, M.L., Allison, S.C., Stanley, C.M., Glenn, S., Weiner, M.W., \& Miller, B.L. (2006). Structural anatomy of empathy in neurodegenerative disease. Brain, 129(Pt 11), 2945-2956.

Rascovsky, K., Salmon, D.P., Lipton, A.M., Leverenz, J.B., DeCarli, C., Jagust, W.J., et al., (2005). Rate of progression differs in frontotemporal dementia and Alzheimer disease. Neurology, 65(3), 397-403.

Reitan, R. (1993). The Halstead-Reitan Neuropsychological Test Battery: Theory and clinical interpretation. Tucson, AZ: Neuropsychology Press.

Rosen, H.J., Allison, S.C., Schauer, G.F., Gorno-Tempini, M.L., Weiner, M.W., \& Miller, B.L. (2005). Neuroanatomical correlates of behavioural disorders in dementia. Brain, 128(Pt 11), 2612-2625.

Rosen, H.J., Gorno-Tempini, M.L., Goldman, W.P., Perry, R.J., Schuff, N., Weiner, M., Feiwell, R., Kramer, J.H., \& Miller, B.L. (2002). Patterns of brain atrophy in frontotemporal dementia and semantic dementia. Neurology, 58(2), 198-208.

Simmonds, D.J., Pekar, J.J., \& Mostofsky, S.H. (2008). Meta-analysis of Go/No-go tasks demonstrating that fMRI activation associated with response inhibition is task-dependent. Neuropsychologia, 46(1), 224-232.

Stokholm, J., Vogel, A., Gade, A., \& Waldemar, G. (2006). Heterogeneity in executive impairment in patients with very mild 
Alzheimer's disease. Dementia and Geriatric Cognitive Disorders, 22(1), 54-59.

Stuss, D.T., Bisschop, S.M., Alexander, M.P., Levine, B., Katz, D., \& Izukawa, D. (2001). The Trail Making Test: A study in focal lesion patients. Psychological Assessment, 13(2), 230-239.

Sullivan, E.V. \& Sagar, H.J. (1991). Double dissociation of shortterm and long-term memory for nonverbal material in Parkinson's disease and global amnesia. A further analysis. Brain, 114(Pt 2), 893-906.

Tabachnick, B.G. \& Fidell, L.S. (2001). Using multivariate statistics (4th ed.). Needham Heights, MA: Allyn and Bacon.

Teri, L., Hughes, J.P., \& Larson, E.B. (1990). Cognitive deterioration in Alzheimer's disease: Behavioral and health factors. Journal of Gerontology, 45(2), P58-P63.

Testa, C., Laakso, M.P., Sabattoli, F., Rossi, R., Beltramello, A., Soininen, H., \& Frisoni, G.B. (2004). A comparison between the accuracy of voxel-based morphometry and hippocampal volum- etry in Alzheimer's disease. Journal of Magnetic Resonance Imaging, 19(3), 274-282.

Tranel, D., Bechara, A., \& Denburg, N.L. (2002). Asymmetric functional roles of right and left ventromedial prefrontal cortices in social conduct, decision-making, and emotional processing. Cortex, 38(4), 589-612.

Viskontas, I.V., Possin, K.L., \& Miller, B.L. (2007). Symptoms of frontotemporal dementia provide insights into orbitofrontal cortex function and social behavior. Annals of the New York Academy of Sciences, 1121, 528-545.

Wallis, J.D., Anderson, K.C., \& Miller, E.K. (2001). Single neurons in prefrontal cortex encode abstract rules. Nature, 411(6840), 953-956.

Wittenberg, D., Possin, K.L., Rascovsky, K., Rankin, K.P., Miller, B.L., \& Kramer, J.H. (2008). The early neuropsychological and behavioral characteristics of frontotemporal dementia. Neuropsychology Review, 18(1), 91-102. 